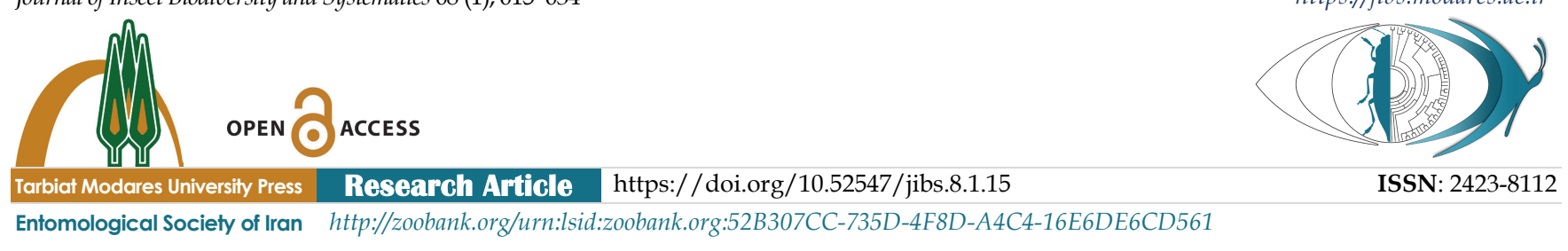

\title{
A checklist of the bees of the genus Hylaeus Fabricius, 1793 (Hymenoptera: Apoidea: Colletidae) of Iran
}

\author{
Mohammad Allahverdi \\ Department of Plant Protection, Faculty of Agriculture, Ferdowsi University of Mashhad, Mashhad, Iran. \\ $\triangle$ allahverdi.md@gmail.com; (ID https://orcid.org/0000-0002-4166-4204
}

\section{Vladimir G. Radchenko}

Institute for Evolutionary Ecology of the National Academy of Sciences of Ukraine, acad. Lebedev, 37, Kiev 03143, Ukraine. هrog@nas.gov.ua; (i) https://orcid.org/0000-0002-8679-1362

\section{Lida Fekrat}

Department of Plant Protection, Faculty of Agriculture, Ferdowsi University of Mashhad, Mashhad, Iran. $\triangle$ fekrat@um.ac.ir; (i) https://orcid.org/0000-0002-5189-9488

\section{Hossein Sadeghi Namaghi}

Department of Plant Protection, Faculty of Agriculture, Ferdowsi University of Mashhad, Mashhad, Iran. $\triangle$ sadeghin@um.ac.ir; (iD https://orcid.org/0000-0002-8329-2699

\begin{tabular}{|c|c|}
\hline & 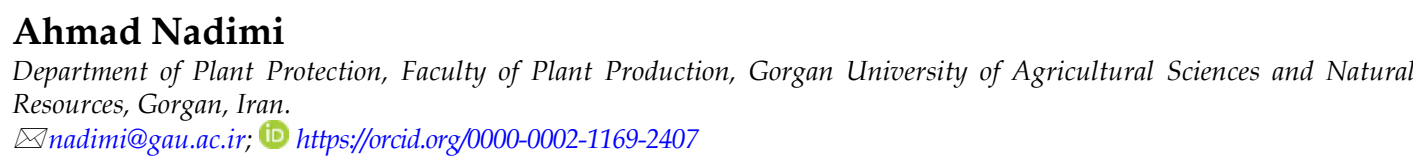 \\
\hline $\begin{array}{l}\text { Received: } \\
16 \text { October, } 2021\end{array}$ & $\begin{array}{l}\text { A B STRACT. A checklist of the bees of the genus Hylaeus Fabricius, } 1793 \text { of Iran, based } \\
\text { on the literature data and also field surveys in northeastern Iran (North Khorasan and } \\
\text { Khorasan-e Razavi provinces) is presented. The resulting checklist comprises nine } \\
\text { subgenera and } 63 \text { species. Four species of the genus Hylaeus, including H. (Lambdopsis) } \\
\text { rinki (Gorski, 1852), H. (Spatulariella) hyalinatus Smith, 1842, H. (Spatulariella) punctatus }\end{array}$ \\
\hline $\begin{array}{l}\text { Accepted: } \\
\text { 13 November, } 2021 \\
\text { Published: } \\
\text { 05 January, } 2022\end{array}$ & $\begin{array}{l}\text { Iran. The later species is considered as "very rare", has only known in Kazakhstan. A re- } \\
\text { description of the male of H. (Prosopis) lionotus together with the illustrations of } \\
\text { morphological characters is given. }\end{array}$ \\
\hline $\begin{array}{l}\text { Subject Editor: } \\
\text { Ali Asghar Talebi }\end{array}$ & Key words: Distribution, Iran, new reports, rare species, faunal records, pollinators \\
\hline
\end{tabular}

\section{INTRODUCTION}

The momentous role of bees, as the pivotal pollinators, in ecosystem stability and food production is undeniable due to their unavoidable contribution in the pollination of both cultivated and wild plants as well as their advanced interactions with flowering plants (Radchenko \& Psenko, 1994, 1996; SteffanDewenter \& Tscharntke, 2001; Huber et al., 2005; Müller et al., 2006). Meticulous exploitation and management of bees, as the foremost pollinators of food crops, are important issues that need to be considered as a matter of priority (Arbuckle et al., 2001; Eardley et al., 2010). The intricacy of bees'

\section{Corresponding author: Fekrat, L., E-mail: fekrat@um.ac.ir}

Copyright (c) 2022, Allahverdi et al. This is an open access article distributed under the terms of the Creative Commons NonCommercial Attribution License (CC BY NC 4.0), which permits Share - copy and redistribute the material in any medium or format, and Adapt - remix, transform, and build upon the material, under the Attribution-NonCommercial terms. 
taxonomy, the minuscule number of trained taxonomists working today, and the lack of comprehensive identification keys as well as limited taxonomic revisions are the main problems, while assessing the biodiversity of these principal pollinators (Francisco et al., 2008). The prominence of bee studies is eminently incontrovertible and has been emphasized in numerous global research projects. Bees, as the keystone species, play a drastic conservative role in the terrestrial ecosystems, but unfortunately some of them are endangered. An increased risk of extinction forces the researchers to survey about the biodiversity of bees as target groups (Sárospataki et al., 2005; Radchenko, 2017). The bees of the world are composed of approximately 20000 species belonging to seven families including: Andrenidae, Apidae, Colletidae, Halictidae, Megachilidae, Melittidae and Stenotritidae (only found in Australia) (Michener, 2007; Ascher \& Pickering, 2021). Among these families, Colletidae, with more than 2700 species, is ranked fifth in terms of number of species. This family usually consists of species with short, truncate or bilobed glossa and is represented in Holarctic region with only two genera including Colletes Latreille, 1802 and Hylaeus Fabricius, 1793 (Michener, 2007; Zhou et al., 2020).

The subfamily Hylaeinae (Hymenoptera: Apoidea: Colletidae) represented by a single genus Hylaeus in the Palaearctic region. Other genera of this subfamily are restricted to Africa, Australia, New Zealand, New Guinea and nearby islands (Michener, 2007). 55 subgenera and 750 species of the genus Hylaeus are documented, worldwide (Michener, 2007; Dathe, 2015; Ascher \& Pickering, 2021), of which 11 subgenera and more than 200 species are currently known from the Palaearctic region (Michener, 2007; Proshchalykin \& Dathe, 2012, 2016). Nine subgenera and 59 species of the genus Hylaeus are so far reported from Iran (Dathe, 1980a; Warncke, 1981; Grace, 2010; Khodaparast \& Monfared, 2012; Kiani Bakiani et al., 2016; Khodarahmi Ghahnavieh \& Monfared, 2019; Ascher \& Pickering, 2021). The first identification key to the subgenera of Hylaeus, as well as the European species of this genus was provided by Dathe (1980b). One of the first specific studies about the geographical distribution and ecology of Hylaeus species was conducted by Koster (1986). Identification keys to Hylaeus species of China, Asian Russia, Central Asia and Turkey were presented by Chen \& Xu (2009), Proshchalykin \& Dathe (2012), Dathe \& Proshchalykin (2018) and Ozbek \& Dathe (2020), respectively. Additionally, Proshchalykin \& Dathe (2021) provided an updated checklist of Hylaeus species of Caucasus region (Armenia, Azerbaijan, Georgia \& Southern Russia). Despite being one of the important centers of bee diversity in the Palaearctic region (Nadimi et al., 2014; Allahverdi et al., 2016), the Iranian bee fauna (with about 900 species) in many taxa is still insufficiently studied (Khaghaninia et al., 2013; Nadimi et al., 2013a, 2013b; Allahverdi et al., 2015; Salarian et al., 2016; Safi et al., 2018; Khodarahmi Ghahnavieh \& Monfared, 2019). With only a few scattered records and descriptions in the literature, the Iranian Colletidae fauna is not an exception (Dathe, 1980b; Izadi et al., 2006; Khodaparast \& Monfared, 2012). The genus Hylaeus is also still poorly known in Iran. The most important data of this taxon in the country came from the publications by Popov (1967), Dathe (1980a) and Warncke (1981) which are all over 35 years old. Out of 52 species of bees of the superfamily Apoidea which were reported by Kiani Bakiani et al. (2016), only 1 new record belonged to the genus Hylaeus. In one of the latest studies on the pollinator bees of the superfamily Apoidea, 14 species of the genus Hylaeus were reported from Isfahan province which among them, four species were introduced to Iran for the first time (Khodarahmi Ghahnavieh \& Monfared, 2019).

In this study, a list of 63 species of Iranian Hylaeus is presented together with notes on their local and general distributions. Moreover, we discuss about a rare species, Hylaeus (Prosopis) lionotus (Alfken, 1909), which has been previously recorded only from Kazakhstan.

\section{MATERIAL AND METHODS}

The list summarizes literature of Iranian Hylaeus species along with some additional information comes from field surveys of several localities in northeastern Iran, between the years 2017 and 2018. Coordinate data were recorded by Garmin eTrex ${ }^{\circledR} 10$ (Garmin (Asia) Corporation, New Taipei, Taiwan). Samples were collected with a sweep net, killed within ethyl acetate and mounted subsequently. Color 
photographs of $H$. lionotus were taken using a Canon ${ }^{\circledR}$ Mark-II 5D and Canon ${ }^{\circledR}$ EOS 5DS R cameras (Canon Inc., Tokyo, Japan) attached to a stereomicroscope with Leica ${ }^{\circledR}$ LED5000 HDI Illuminator by the second author in the IEENASU. Other photographs were made using a Canon ${ }^{\circledR}$ EOS REBEL XS attached to Olympus ${ }^{\circledR}$ stereomicroscope in the Entomology Laboratory of Ferdowsi University of Mashhad. Both classification and nomenclature followed Michener (2007) and Ascher \& Pickering (2021). Samples were identified to the species level using relevant identification keys (Dathe, 1980b; Koster, 1986; Ozbek \& Dathe, 2020). The morphological terminology for the re-description of the species follows Michener (2007). The map of Hylaeus species found in the studied area was generated in ArcMap $^{\circledR}$ 10.6.1 software (Fig. 1). Examined specimens were deposited in the insect collection of Ferdowsi University of Mashhad, Iran, except H. lionotus which is stored in the collection of Institute for Evolutionary Ecology of the National Academy of Sciences of Ukraine (IEENASU, Kiev).

\section{RESULTS}

In this study, nine Hylaeinae species belonging to five subgenera of Hylaeus, including Hylaeus Fabricius 1793, Lambdopsis Popov, 1939, Koptogaster Alfken, 1912, Prosopis Fabricius, 1804 and Spatulariella Popov, 1939, were collected and identified, among them, four species are new to the fauna of Iran. The species are presented alphabetically. List of previously known species of Hylaeus, as well as newly recorded species (marked with an asterisk, ${ }^{*}$ ) are presented here.

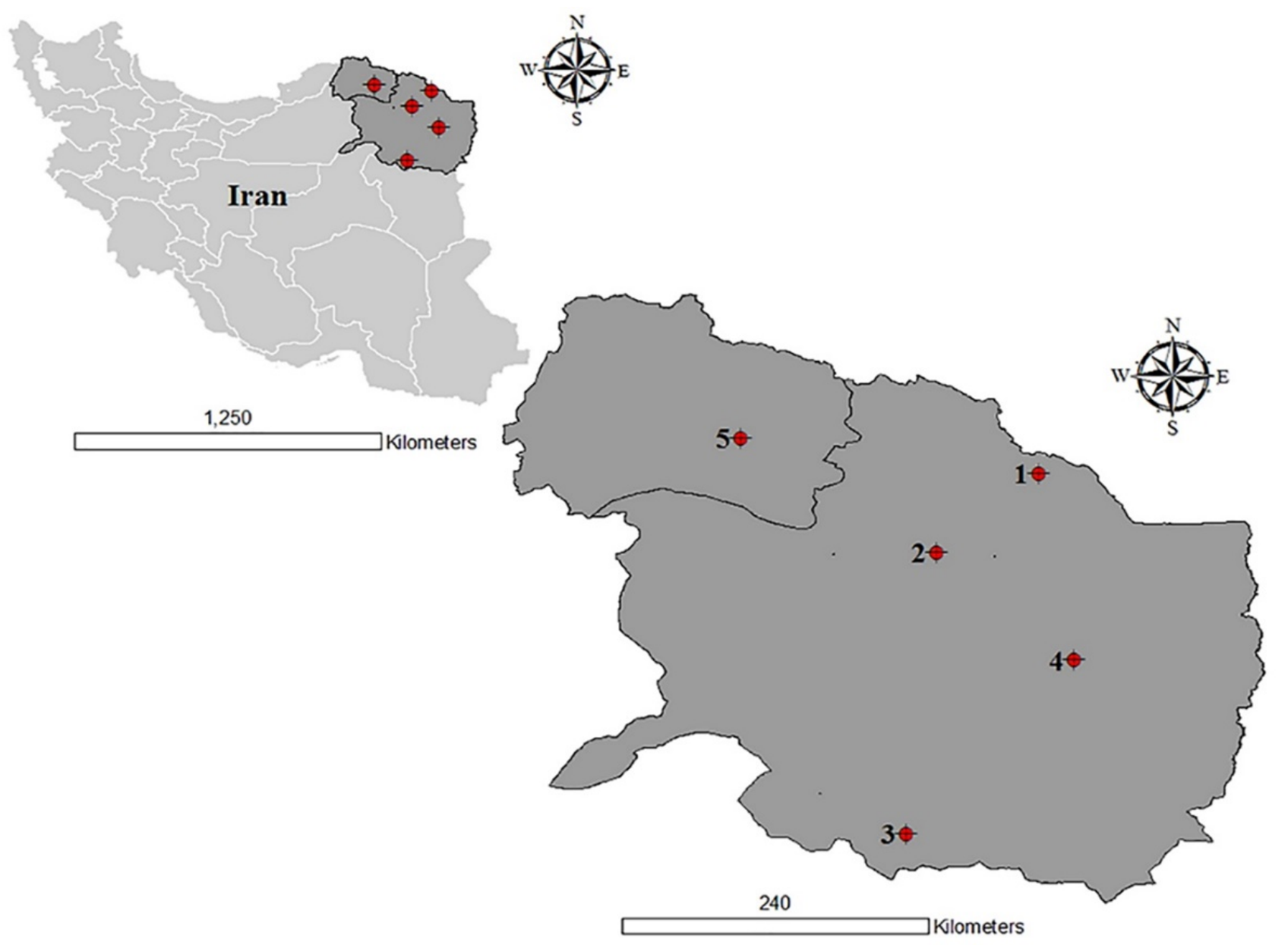

Figure 1. Sampling points of Hylaeus species found in the studied area, northeastern Iran: 1. Khorasan-e Razavi, Qare su, 2. Khorasan-e Razavi, Neishabour, Bujan, 3. Khorasan-e Razavi, Gonabad, 4. Khorasan-e Razavi, Fariman Dam, 5. Northern Khorasan, Shirvan, Gelian. 
Checklist of Hylaeus species in Iran

Subgenus: Abrupta Méhelÿ, 1935

Type species: Hylaeus cornutus Curtis, 1831

Hylaeus (Abrupta) cornutus Curtis, 1831

Distribution in Iran: Golestan (Warncke, 1981), Hamadan, Isfahan (Khodarahmi Ghahnavieh \& Monfared, 2019).

General distribution: Eastern (Turkmenistan) and Western (Azerbaijan, Cyprus, Denmark, France, Germany, Greece, Iran, Italy, Russia, Slovakia, Spain, Syria, Tunisia, Turkey, Ukraine, United Kingdom) Palaearctic (Khodarahmi Ghahnavieh \& Monfared, 2019; Ascher \& Pickering, 2021).

Subgenus: Dentigera Popov, 1939

Type species: Hylaeus brevicornis Nylander, 1852

Hylaeus (Dentigera) acer Dathe, 1980

Distribution in Iran: Mazandaran (Warncke, 1981; Ascher \& Pickering, 2021).

General distribution: Western Palaearctic (Iran, Turkey) (Ascher \& Pickering, 2021).

Hylaeus (Dentigera) brevicornis Nylander, 1852

Distribution in Iran: Alborz, Fars, Golestan, Hamadan, Kerman, Khorasan-e Razavi, Mazandaran (Warncke, 1981), Isfahan (Khodarahmi Ghahnavieh \& Monfared, 2019).

General distribution: Eastern (Afghanistan) and Western (Czech Republic, Denmark, Estonia, Finland, France, Georgia, Greece, Ireland, Jordan, Italy, Latvia, Norway, Portugal, Romania, Russia, Slovakia, Spain, Sweden, Switzerland, Turkey, Uzbekistan, Iran) Palaearctic (Khodarahmi Ghahnavieh \& Monfared, 2019; Ascher \& Pickering, 2021).

Hylaeus (Dentigera) gredleri Förster, 1871

Distribution in Iran: Fars (Kiani Bakiani et al., 2016); No locality cited (Warncke, 1981).

General distribution: Eastern (Kazakhstan) and Western (Austria, Azerbaijan, France, Germany, Greece, Hungary, Italy, Lebanon, Lithuania, Morocco, Russia, Spain, Tunisia, Turkey, Iran) Palaearctic (Kiani Bakiani et al., 2016; Ascher \& Pickering, 2021).

Hylaeus (Dentigera) imparilis Förster, 1871

Distribution in Iran: Isfahan (Khodarahmi Ghahnavieh \& Monfared, 2019).

General distribution: Western Palaearctic (Algeria, Azerbaijan, Bulgaria, Cyprus, France, Greece, Hungary, Iran, Italy, Spain, Switzerland, Tunisia, Turkey) (Khodarahmi Ghahnavieh \& Monfared, 2019; Ascher \& Pickering, 2021).

\section{Hylaeus (Dentigera) kahri Förster, 1871}

Distribution in Iran: No locality cited (Warncke, 1981).

General distribution: Western Palaearctic (Algeria, Azerbaijan, Germany, France, Greece, Iran, Italy, Luxembourg, Turkey, Slovakia, Spain, Syria) (Ascher \& Pickering, 2021).

Hylaeus (Dentigera) punctus Förster, 1871

Distribution in Iran: Fars (Kiani Bakiani et al., 2016); No locality cited (Warncke, 1981). 
General distribution: Western Palaearctic (Bulgaria, Croatia, Greece, Iran, Israel, Turkey) (Kiani Bakiani et al., 2016; Ascher \& Pickering, 2021).

Subgenus: Hylaeus Fabricius s. str.

Type species: Prosopis annulata Fabricius, 1804

Hylaeus (Hylaeus) angustatus (Schenck, 1861)

Distribution in Iran: No locality cited (Warncke, 1981).

General distribution: Eastern (China, Mongolia) and Western (Armenia, Czech Republic, Finland, France, Georgia, Greece, Iran, Italy, Jordan, Morocco, Norway, Portugal, Russia, Sweden, Switzerland, Turkey, Ukraine) Palaearctic (Ascher \& Pickering, 2021).

Hylaeus (Hylaeus) araxanus (Warncke, 1981)

Distribution in Iran: Alborz, Fars, Hamadan, Kerman, Sistan-o Balouchistan, South Khorasan (Warncke, 1981), Isfahan (Khodarahmi Ghahnavieh \& Monfared, 2019).

General distribution: Western Palaearctic (Iran, Turkey) (Khodarahmi Ghahnavieh \& Monfared, 2019; Ascher \& Pickering, 2021).

Hylaeus (Hylaeus) cervinus (Warncke, 1992)

Distribution in Iran: No locality cited (Warncke, 1981).

General distribution: Western Palaearctic (Iran, Turkey) (Ascher \& Pickering, 2021).

Hylaeus (Hylaeus) communis Nylander, 1852

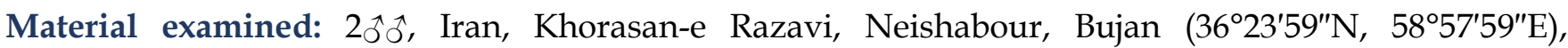
17.IV.2017, Leg.: M. Allahverdi.

Food association: Matricaria chamomilla Linnaeus (Asteraceae).

Distribution in Iran: Isfahan, Mazandaran (Warncke, 1981).

General distribution: Eastern (Afghanistan, China, Estonia, Kazakhstan, Turkmenistan, Tajikistan) and Western (Armenia, Azerbaijan, Canada, Czech Republic, Denmark, England, Finland, France, Georgia, Greece, Iran, Ireland, Italy, Norway, Portugal, Romania, Russia, Slovakia, Spain, Sweden, Switzerland, Tunisia, Turkey) Palaearctic (Ascher \& Pickering, 2021).

Hylaeus (Hylaeus) crispulus Dathe, 1980

Distribution in Iran: Mazandaran (Warncke, 1981).

General distribution: Western Palaearctic (Iran, Israel, Turkey) (Ascher \& Pickering, 2021).

Hylaeus (Hylaeus) dolichocephalus Morawitz, 1876

Distribution in Iran: Isfahan (Khodarahmi Ghahnavieh \& Monfared, 2019), South Khorasan (Warncke, 1981). General distribution: Eastern (Afghanistan, China, Kazakhstan, Mongolia, Tajikistan) and Western (Iran, Turkey) Palaearctic (Khodarahmi Ghahnavieh \& Monfared, 2019; Ascher \& Pickering, 2021).

Hylaeus (Hylaeus) ebmeri Dathe, 1980

Distribution in Iran: No locality cited (Warncke, 1981; Ascher \& Pickering, 2021).

General distribution: Western Palaearctic (Iran) (Warncke, 1981; Ascher \& Pickering, 2021). 
Hylaeus (Hylaeus) fedtschenkoi (Cockerell, 1906)

Distribution in Iran: No locality cited (Warncke, 1981).

General distribution: Eastern (Afghanistan, China, Mongolia, Uzbekistan) and Western (Iran) Palaearctic (Ascher \& Pickering, 2021).

Hylaeus (Hylaeus) hungaricus (Alfken, 1905)

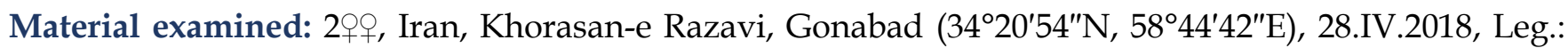
M. Allahverdi.

Food association: Tamarix sp. (Tamaricaceae).

Distribution in Iran: Golestan (Warncke, 1981).

General distribution: Eastern (Afghanistan, Kyrgyzstan, Mongolia, Tajikistan, Turkmenistan) and Western (Iran, Georgia, Russia) Palaearctic (Ascher \& Pickering, 2021).

Hylaeus (Hylaeus) implicatus Dathe, 1980

Distribution in Iran: Isfahan (Khodarahmi Ghahnavieh \& Monfared, 2019).

General distribution: Western Palaearctic (Iran, Turkey) (Khodarahmi Ghahnavieh \& Monfared, 2019; Ascher \& Pickering, 2021).

Hylaeus (Hylaeus) jantaris Dathe, 1980

Distribution in Iran: No locality cited (Warncke, 1981).

General distribution: Western Palaearctic (Iran, Turkey) (Ascher \& Pickering, 2021).

Hylaeus (Hylaeus) kotschisus (Warncke, 1981)

Distribution in Iran: Isfahan (Khodarahmi Ghahnavieh \& Monfared, 2019), Khorasan-e Razavi, Mazandaran (Warncke, 1981).

General distribution: Western Palaearctic (Iran, Turkey) (Khodarahmi Ghahnavieh \& Monfared, 2019; Ascher \& Pickering, 2021).

Hylaeus (Hylaeus) leptocephalus (Morawitz, 1871)

Distribution in Iran: Alborz, Hamadan, Kurdistan, Zanjan (Warncke, 1981), Isfahan (Khodarahmi Ghahnavieh and Monfared, 2019).

General distribution: Nearctic (Canada, United States) and Western Palaearctic (Armenia Bulgaria, Czech Republic, Denmark, Germany, Greece, Iran, Italy, Latvia, Spain, Turkey, Ukraine) (Ascher \& Pickering, 2021).

Hylaeus (Hylaeus) moricei (Friese, 1898)

Distribution in Iran: Alborz, Hamadan (Warncke, 1981), Isfahan (Khodarahmi Ghahnavieh \& Monfared, 2019).

General distribution: Western Palaearctic (Armenia, Austria, Egypt, Germany, Greece, Iran, Italy, Jordan, Lithuania, Russia, Spain, Turkey, Ukraine) (Khodarahmi Ghahnavieh \& Monfared, 2019; Ascher \& Pickering, 2021).

Hylaeus (Hylaeus) nigrifacies Bramson, 1879

Distribution in Iran: Alborz, Hamadan, Qazvin (Warncke, 1981).

General distribution: Western Palaearctic (Iran, Ukraine) (Ascher \& Pickering, 2021). 
Hylaeus (Hylaeus) nigritus (Fabricius, 1798)

Distribution in Iran: No locality cited (Warncke, 1981).

General distribution: Eastern (Kazakhstan) and Western (Austria, Belarus, Estonia, Finland, Georgia, Greece, Germany, Iran, Italy, Morocco, Russia, Spain, Turkey, Ukraine) Palaearctic (Ascher \& Pickering, 2021).

Hylaeus (Hylaeus) scutellaris Morawitz, 1874

Distribution in Iran: Alborz (Warncke, 1981; Ascher \& Pickering, 2021), Isfahan (Khodarahmi Ghahnavieh and Monfared, 2019).

General distribution: Western Palaearctic (Azerbaijan, Cyprus, Greece, Iran, Slovakia, Turkey) (Khodarahmi Ghahnavieh \& Monfared, 2019; Ascher \& Pickering, 2021).

Hylaeus (Hylaeus) tardus (Warncke, 1981)

Distribution in Iran: Hamadan, Fars (Warncke, 1981), Isfahan (Khodarahmi Ghahnavieh \& Monfared, 2019).

General distribution: Western Palaearctic (Azerbaijan, Iran, Turkey) (Khodarahmi Ghahnavieh \& Monfared, 2019; Ascher \& Pickering, 2021).

Hylaeus (Hylaeus) trifidus (Alfken, 1936)

Distribution in Iran: Isfahan (Warncke, 1981).

General distribution: Western Palaearctic (Algeria, Greece, Iran, Israel, Turkey) (Ascher \& Pickering, 2021).

Hylaeus (Hylaeus) trisignatus Morawitz, 1876

Distribution in Iran: Isfahan (Khodarahmi Ghahnavieh \& Monfared, 2019).

General distribution: Eastern (Tajikistan) and Western (Iran, Turkey) Palaearctic (Khodarahmi Ghahnavieh \& Monfared, 2019; Ozbek \& Dathe, 2020; Ascher \& Pickering, 2021).

Hylaeus (Hylaeus) vulgaris Morawitz, 1876

Distribution in Iran: Fars, Khorasan (Warncke, 1981).

General distribution: Eastern (Afghanistan, China, Kazakhstan, Mongolia, Tajikistan) and Western (Iran) Palaearctic (Ascher \& Pickering, 2021).

Subgenus: Koptogaster Alfken, 1912

Type species: Prosopis bifasciata Jurine, 1807

Hylaeus (Koptogaster) bifasciatus (Jurine, 1807)

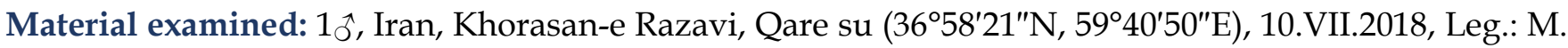
Allahverdi.

Food association: Asteraceae.

Distribution in Iran: East Azarbaijan (Warncke, 1981).

General distribution: Western Palaearctic (Albania, Belgium, Georgia, Germany, Greece, Iran, Israel, Italy, Malaysia, Romania, Turkey, Ukraine) (Ascher \& Pickering, 2021).

Hylaeus (Koptogaster) punctulatissimus Smith, 1842

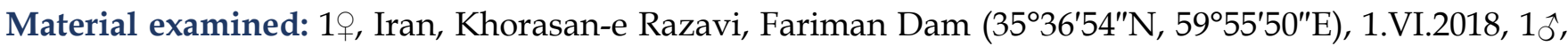
Leg.: M. Allahverdi. 
Food association: Astragalus gossypinus (Fabaceae).

Distribution in Iran: Mazandaran (Warncke, 1981).

General distribution: Western Palaearctic (Bulgaria, Czech Republic, Denmark, Georgia, Germany, Greece, Iran, Israel, Italy, Moldova, Morocco, Netherland, Portugal, Russia, Slovakia, Switzerland, Turkey, Ukraine) (Ascher \& Pickering, 2021).

Subgenus: Lambdopsis Popov, 1939

Type species: Melitta annularis Kirby, 1802

Hylaeus (Lambdopsis) annularis (Kirby, 1802)

Distribution in Iran: Golestan, Mazandaran, North Khorasan (Warncke, 1981).

General distribution: Eastern (Tajikistan) and Western (Algeria, Belarus, Bulgaria, Czech Republic, Denmark, England, Finland, France, Germany, Iran, Italy, Netherlands, Russia, Spain, Sweden, Tunisia) Palaearctic (Ascher \& Pickering, 2021).

Hylaeus (Lambdopsis) dilatatus (Kirby, 1802)

Distribution in Iran: No locality cited (Warncke, 1981).

General distribution: Eastern (Kazakhstan) and Western (Albania, Algeria, Armenia, Austria, Azerbaijan, Denmark, England, Finland, Greece, Iran, Italy, Lithuania, Portugal, Russia, Switzerland, Tunisia, Turkey, Ukraine) Palaearctic (Ascher \& Pickering, 2021).

Hylaeus (Lambdopsis) sariensis Dathe, 1980

Distribution in Iran: Mazandaran (Warncke, 1981; Ascher \& Pickering, 2021).

General distribution: Western Palaearctic (Iran) (Warncke, 1981; Ascher \& Pickering, 2021).

Hylaeus (Lambdopsis) scutellatus (Spinola, 1838)

Distribution in Iran: Alborz (Khodarahmi Ghahnavieh \& Monfared, 2019; Ascher \& Pickering, 2021).

General distribution: Western Palaearctic (Azerbaijan, Cyprus, Greece, Iran, Slovakia, Turkey) (Khodarahmi Ghahnavieh \& Monfared, 2019; Ascher \& Pickering, 2021).

Hylaeus (Lambdopsis) rinki (Górski, 1852)* (Figs. 3A-B)

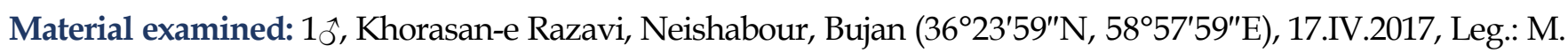

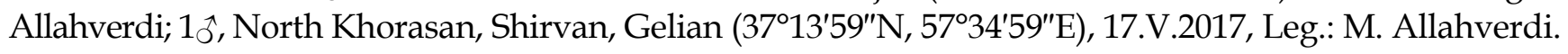

Food association: Astragalus gossypinus (Fabaceae) and unknown for second locality.

General distribution: Western Palaearctic (Austria, Belgium, China, Estonia, Finland, France, Germany, Iran (New record), Italy, Latvia, Mongolia, Russia, Slovakia, Sweden, Switzerland, Ukraine) (Ascher \& Pickering, 2021).

Subgenus: Paraprosopis Popov, 1939

Type species: Hylaeus pictipes Nylander, 1852

Hylaeus (Paraprosopis) albonotatus (Walker, 1871)

Distribution in Iran: Kerman, Khuzestan (Warncke, 1981).

General distribution: Afrotropical (United Arab Emirates) and Western Palaearctic (Chad, Djibouti, Egypt, Iran, Israel, Libya, Mauritania, Morocco, Sudan, Tunisia, Yemen) (Ascher \& Pickering, 2021). 
Hylaeus (Paraprosopis) lineolatus (Schenck, 1861)

Distribution in Iran: Alborz, Bushehr, Fars, Kerman, Mazandaran (Warncke, 1981).

General distribution: Western Palaearctic (Azerbaijan, Cyprus, Czech Republic, Germany, Greece, Iran, Italy, Moldova, Portugal, Russia, Slovakia Spain, Turkey, Ukraine) (Ascher \& Pickering, 2021).

Hylaeus (Paraprosopis) sinuatus (Schenck, 1853)

Distribution in Iran: Bushehr, Hamadan (Warncke, 1981).

General distribution: Western Palaearctic (Denmark, France, Germany, Iran, Lithuania, Portugal, Russa, Slovakia, Sweden, Switzerland, Turkey, Ukraine) (Ascher \& Pickering, 2021).

Hylaeus (Paraprosopis) socheri Dathe, 2010

Distribution in Iran: Yazd (Warncke, 1981; Ascher \& Pickering, 2021).

General distribution: Western Palaearctic (Iran (Warncke, 1981; Ascher \& Pickering, 2021), Turkey (Ozbek \& Dathe, 2020)).

Hylaeus (Paraprosopis) styriacus Förster, 1871

Distribution in Iran: No locality cited (Warncke, 1981).

General distribution: Western Palaearctic (Azerbaijan, Czech Republic, Estonia, Germany, Georgia, Greece, Iran, Italy, Lebanon, Moldova, Poland, Russia, Turkey) (Ascher \& Pickering, 2021).

Subgenus: Patagiata Blüthgen, 1949

Type species: Prosopis difformis Eversmann, 1852

Hylaeus (Patagiata) difformis (Eversmann, 1852)

Distribution in Iran: Golestan (Warncke, 1981).

General distribution: Eastern (China) and Western (Denmark, Georgia, Greece, Finland, France, Iran, Italy, Latvia, Macedonia, Morocco, Poland, Russia, Spain, Sweden, Turkey, Ukraine) Palaearctic (Ascher \& Pickering, 2021).

Subgenus: Prosopis Fabricius, 1804

Type species: Mellinus bipunctatus Fabricius, 1798

Hylaeus (Prosopis) damascenus (Magretti, 1890)

Distribution in Iran: No locality cited (Warncke, 1981).

General distribution: Western Palaearctic (Iran, Iraq, Israel, Macedonia, Turkey) (Ascher \& Pickering, 2021).

Hylaeus (Prosopis) excelsus (Alfken, 1931)

Distribution in Iran: Golestan, Mazandaran (Warncke, 1981), Isfahan (Khodarahmi Ghahnavieh \& Monfared, 2019).

General distribution: Western Palaearctic (Iran, Turkey) (Khodarahmi Ghahnavieh \& Monfared, 2019; Ascher \& Pickering, 2021).

Hylaeus (Prosopis) gibbus Saunders, 1850

Distributionin in Iran: Golestan, Guilan, Hamadan, Markazi, Mazandaran (Warncke, 1981). 
General distribution: Eastern (Kazakhstan) and Western (Austria, Azerbaijan, Belarus, Cyprus, Finland, Georgia, Germany, Greece, Iran, Iraq, Netherland, Russia, Spain, Sweden, Turkey, Ukraine) Palaearctic (Ascher \& Pickering, 2021).

Hylaeus (Prosopis) hyrcanius Dathe, 1980

Distribution in Iran: Mazandaran (Warncke, 1981).

General distribution: Western Palaearctic (Azerbaijan, Iran, Russia) (Ascher \& Pickering, 2021).

Hylaeus (Prosopis) laevithorax (Alfken, 1924)

Distribution in Iran: Fars, Kerman, Sistan-o Balouchistan (Warncke, 1981).

General distribution: Eastern (Pakistan) and Western (Iran, Israel) Palaearctic (Ascher \& Pickering, 2021).

Hylaeus (Prosopis) lionotus (Alfken, 1909)*

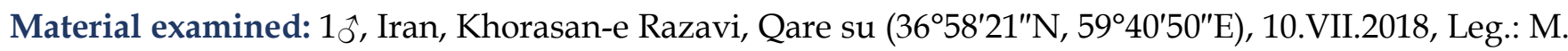
Allahverdi.

Redescription - Based on the non-type material collected from Khorasan-e Razavi.

Male: Total body length $6.0 \mathrm{~mm}$ (Figs. 2A-B).

Head: Width: $1.88 \mathrm{~mm}$; length: $2.10 \mathrm{~mm}$; Scape of antennae expanded, longer than wide, scape more than 2/3 yellow and black at upper side; Flagellum dark brown but antennomeres III to X reddishyellow at lower parts; Clypeus, supraclypeal and two paraocular areas pale-yellow colored, mask completed (Fig. 2F); Clypeus with sparse erect light hairs, supraclypeal area and paraocular area sparsely punctuated, punctuation of supraclypeal area surface at lower sides denser than upper sides; Frons punctuated contiguously; Malar area shortened; Gena punctuated and ridged; Occiput rounded.

Mesosoma: Coloration black, except pronotum with a narrow yellow stripe, axillae, tegula and pronotal lobe pale-yellow, scutum and scutellum glossy, moderately punctuated (Fig. 2B); The integument of thorax completely shiny and glossy; Mesopleura with conspicuously coarse punctuation, total surface of mesosoma with short erect light yellow hairs except propodeal triangle; Propodeal triangle shinyglossy with subcontiguous punctuation and without any hairs and pointed at lower part; Propodeum rugose; Legs black-brown but yellow at basitarsus and basitibia (Fig. 2A), Wings light brown and venation dark brown.

Metasoma: Abdomen coloration very dark but marginal parts of terga more light-reddish at least $1 / 2$ of T1 which expanded in middle of disk, and semitransparent light-yellow on other terga with brown-red premarginal line (Fig. 2E); lateral parts of T1 with distinct long white fringes but in other terga less distinct with short light hairs on marginal zones of terga, T1 and following terga shiny with high subcontiguous coarse punctuation; all terga distinctly, finely and densely punctuated (Figs. 2A, 2B, 2D, 2); Genital capsule with enlarged gonocoxites, gonostylus elongated and rounded apically with impression at the distal part, and sparse long curved bristles (Fig. 2C).

Food association: Asteraceae.

General distribution: Eastern (Kazakhstan) and Western (Iran-New record) Palaearctic (Dathe \& Proshchalykin, 2018; Ascher \& Pickering, 2021).

Hylaeus (Prosopis) maculatus (Alfken, 1904)

Distribution in Iran: No locality cited (Warncke, 1981).

General distribution: Western Palaearctic (Algeria, Iran, Israel, Turkey) (Ascher \& Pickering, 2021). 


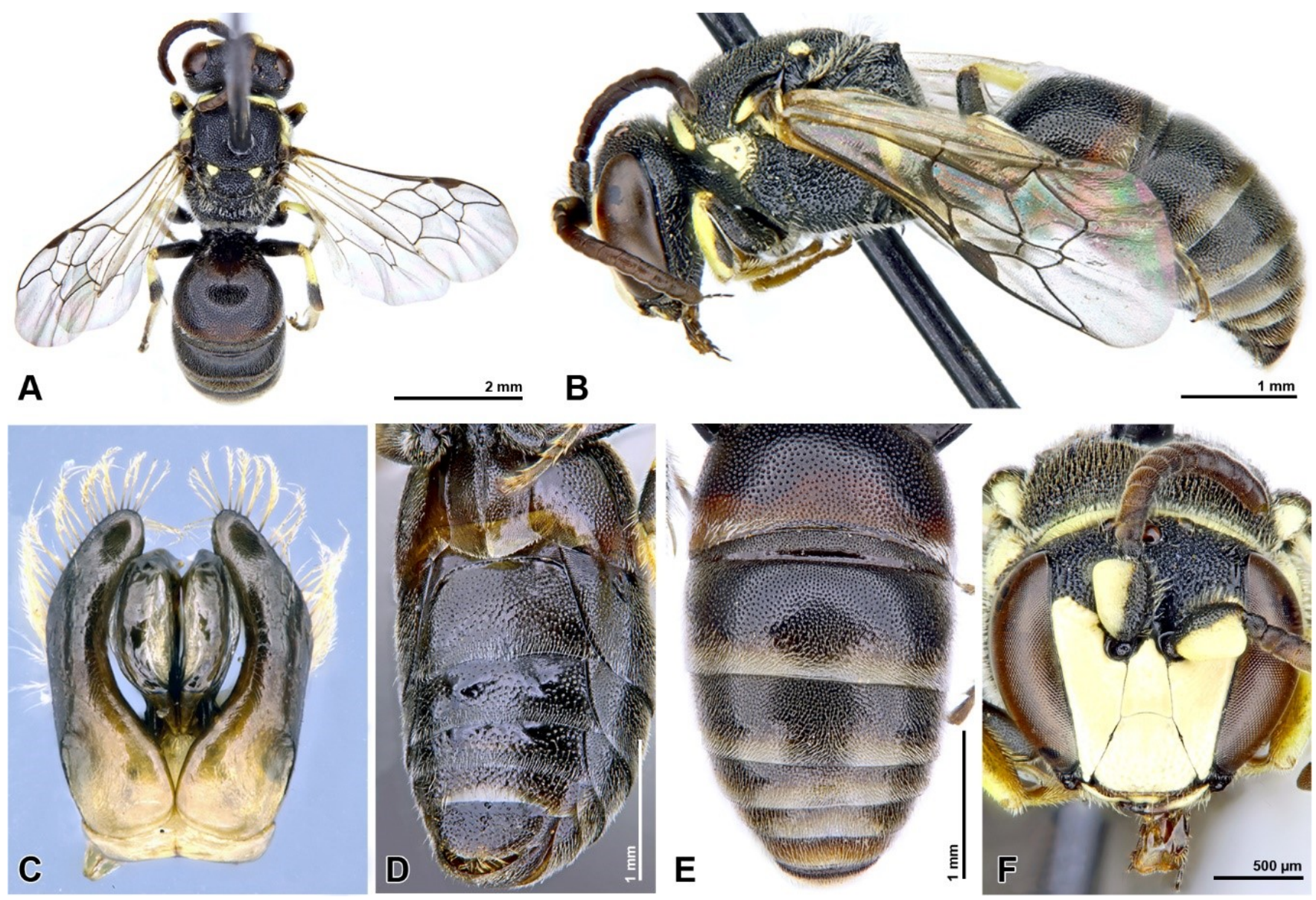

Figure 2. Hylaeus (Prosopis) lionotus (Alfken, 1909): A-B. Total body, C. Genitalia, D-E. Metasoma in ventral and dorsal view, F. Head in frontal view.

\section{Hylaeus (Prosopis) meridionalis Förster, 1871}

Distribution in Iran: Alborz (Warncke, 1981).

General distribution: Eastern (Kyrgyzstan, Tajikistan) and Western (Azerbaijan, Bulgaria, Croatia, Greece, Iran, Italy, Morocco, Portugal, Russia, Switzerland, Tunisia, Turkey, Ukraine) Palaearctic (Ascher \& Pickering, 2021).

Hylaeus (Prosopis) pictus (Smith, 1853)

Distribution in Iran: Isfahan (Khodarahmi Ghahnavieh \& Monfared, 2019).

General distribution: Western Palaearctic (Algeria, Azerbaijan, Cyprus, Egypt, France, Greece, Iran, Italy, Slovakia, Spain, Tunisia, Turkey) (Khodarahmi Ghahnavieh \& Monfared, 2019; Ascher \& Pickering, 2021).

Hylaeus (Prosopis) rubosus (Warncke, 1981)

Distribution in Iran: No locality cited (Warncke, 1981).

General distribution: Palaearctic Western (Iran, Turkey) (Ascher \& Pickering, 2021).

\section{Hylaeus (Prosopis) rugicollis Morawitz, 1874}

Distribution in Iran: Alborz, Azarbaijan, Bushehr, Fars, Guilan, Kerman, Khuzestan (Warncke, 1981). General distribution: Western Palaearctic (Azerbaijan, Greece, Iran, Lebanon, Turkey) (Ascher \& Pickering, 2021). 
Hylaeus (Prosopis) signatus (Panzer, 1798)

Distribution in Iran: No locality cited (Warncke, 1981).

General distribution: Eastern (Kyrgyzstan, Uzbekistan, Turkmenistan) and Western (Azerbaijan, Belarus, Finland, France, Germany, Greece, Iran, Israel, Italy, Morocco, Portugal, Russia, Slovakia, Spain, Sweden, Tunisia, Turkey, Ukraine, United Kingdom) Palaearctic (Ascher \& Pickering, 2021).

Hylaeus (Prosopis) variegatus (Fabricius, 1798)

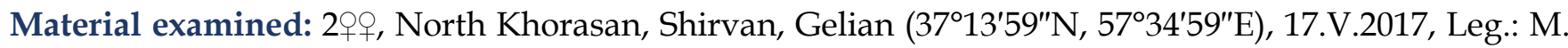
Allahverdi.

Food association: Unknown.

Distribution in Iran: Alborz, Azarbaijan, Bushehr, Fars, Golestan, Hamadan, Kurdistan, Khorasan-e Razavi, Mazandaran, Qazvin, Tehran (Warncke, 1981; Ascher \& Pickering, 2021).

General distribution: Eastern (Afghanistan, China, Kazakhstan, Kyrgyzstan, Tajikistan, Turkmenistan, Uzbekistan) and Western (Albania, Algeria, Armenia, Austria, Bulgaria, Croatia, Czech Republic, Egypt, France, Georgia, Germany, Greece, Iran, Israel, Italy, Jordan, Lebanon, Macedonia, Moldova, Mongolia, Montenegro, Morocco, Netherland, Poland, Portugal, Romania, Russia, Slovakia, Spain, Switzerland, Syria, Tunisia, Turkey, Ukraine) Palaearctic (Ascher \& Pickering, 2021).

Subgenus: Spatulariella Popov, 1939

Type species: Hylaeus hyalinatus Smith, 1842

Hylaeus (Spatulariella) alticola (Warncke, 1981)

Distribution in Iran: Mazandaran (Warncke, 1981; Ascher \& Pickering, 2021).

General distribution: Western Palaearctic (Iran) (Warncke, 1981; Ascher \& Pickering, 2021).

Hylaeus (Spatulariella) armeniacus (Warncke, 1981)

Distribution in Iran: Fars, Tehran (Warncke, 1981).

General distribution: Westeran Palaearctic (Armenia, Iran, Lebanon, Turkey) (Ascher \& Pickering, 2021).

Hylaeus (Spatulariella) iranicus Dathe, 1980

Distribution in Iran: Mazandaran (Warncke, 1981).

General distribution: Western Palaearctic (Iran, Turkey) (Ascher \& Pickering, 2021).

Hylaeus (Spatulariella) irritans Dathe, 1980

Distribution in Iran: No locality cited (Warncke, 1981).

General distribution: Western Palaearctic (Iran, Turkey) (Ascher \& Pickering, 2021).

Hylaeus (Spatulariella) longimaculus (Alfken, 1936)

Distribution in Iran: Hamadan, Tehran (Warncke, 1981; Ascher \& Pickering, 2021).

General distribution: Western Palaearctic (Armenia, Greece, Iran, Israel, Lebanon, Morocco, Turkey) (Ascher \& Pickering, 2021).

Hylaeus (Spatulariella) hyalinatus Smith, 1842* (Figs. 3C-D)

Material examined: 1ㅇ, Iran, Khorasan-e Razavi, Neishabour, Bujan (36²3'59"N, 5857'59"E), 17.IV.2017, Leg.: M. Allahverdi.

Food association: Matricaria chamomilla (Asteraceae). 
General distribution: Western Palaearctic (Belgium, Czech Republic, Denmark, Estonia, Finland, Georgia, Germany, Greece, Iran (New record), Israel, Italy, Ireland, Malaysia, Morocco, Norway, Poland, Portugal, Russia, Spain, Sweden, Turkey, Ukraine, United Kingdom) and Nearctic (Canada, United State) (Ascher \& Pickering, 2021).

Hylaeus (Spatulariella) punctatus (Brullé, 1832)* (Figs. 3E-F)

Material examined: $1 \widehat{\delta}^{\wedge}$, Iran, Khorasan-e Razavi, Gonabad ( $\left.34^{\circ} 20^{\prime} 54^{\prime \prime} \mathrm{N}, 5^{\circ} 44^{\prime} 42^{\prime \prime} \mathrm{E}\right), 28 . I V .2018$, Leg.: M. Allahverdi.

Food association: Tamarix sp. (Tamaricaceae).

General distribution: Western Palaearctic (Azerbaijan, Germany, Greece, Iran (New record), Italy, France, Lebanon, Malta, Poland, Portugal, Romania, Turkey, Ukraine), Nearctic (Argentina, Canada, Chile, United State) (Ascher \& Pickering, 2021).

\section{Unclassified species}

Hylaeus clusius (Warncke, 1981)

Distribution in Iran: Khorasan-e Razavi (Warncke, 1981; Ascher \& Pickering, 2021).

General distribution: Western Palaearctic (Iran) (Warncke, 1981; Ascher \& Pickering, 2021).

Hylaeus oriole (Warncke, 1981)

Distribution in Iran: Mazandaran (Warncke, 1981; Ascher \& Pickering, 2021).

General distribution: Western Palaearctic (Iran) (Warncke, 1981; Ascher \& Pickering, 2021).

\section{DISCUSSION}

Ascher \& Pickering (2021) presented a checklist of 761 world species of the genus Hylaeus including 52 species from Iran. Taking into account the new records of the current study and also considering all new and old local literatures (Warncke, 1981; Grace, 2010; Khodaparast \& Monfared, 2012; Kiani Bakiani et al., 2016; Khodarahmi Ghahnavieh \& Monfared, 2019), the total number of Iranian Hylaeus species reaches to 63. As shown in Fig. 4, up to now nine subgenera of the genus Hylaeus have been reported from Iran. Among all these subgenera, Hylaeus is the most diverse one with 21 species (Fig. 4). This is also the most diverse subgenus of genus Hylaeus in Palaearctic region (Ascher \& Pickering, 2021).

The number of newly recorded species in the current study could be mirrored the high diversity of this bee group in the country. The percentage of Hylaeus species hitherto reported from Iran is $6.5 \%$ of the total species of the genus recorded so far in the world. This percentage is also nearly similar or higher than those of some neighboring countries (Iraq 6.9\%, Azerbaijan 6.6\%, Turkey 4.5\%, Armenia $3.3 \%$, Afghanistan 2.3\%, Turkmenistan 2\% and Pakistan 0.9\%) (Ascher \& Pickering, 2021). It is noteworthy to note that some of these low percentages might be an outcome of inadequate surveys in these countries, rather than being a reflection of the real number of the existed Hylaeus species. In some neibouring countries of Iran such as Afghanistan and Iraq, political instability as well as civil wars probably have had a strong negative effect on taxonomic studies of various groups of insects including bees. In addition to $H$. lionotus, which was recorded as a very rare species by Dathe \& Proshchalykin (2018), two other new reports of this study, i.e. H. rinki and H. punctatus, are also considered as rare species (Celary, 1999; Feitz et al., 2003; Kowalczyk et al., 2009; Haris, 2015). Hylaeus lionotus belongs to the "variegatus group". 

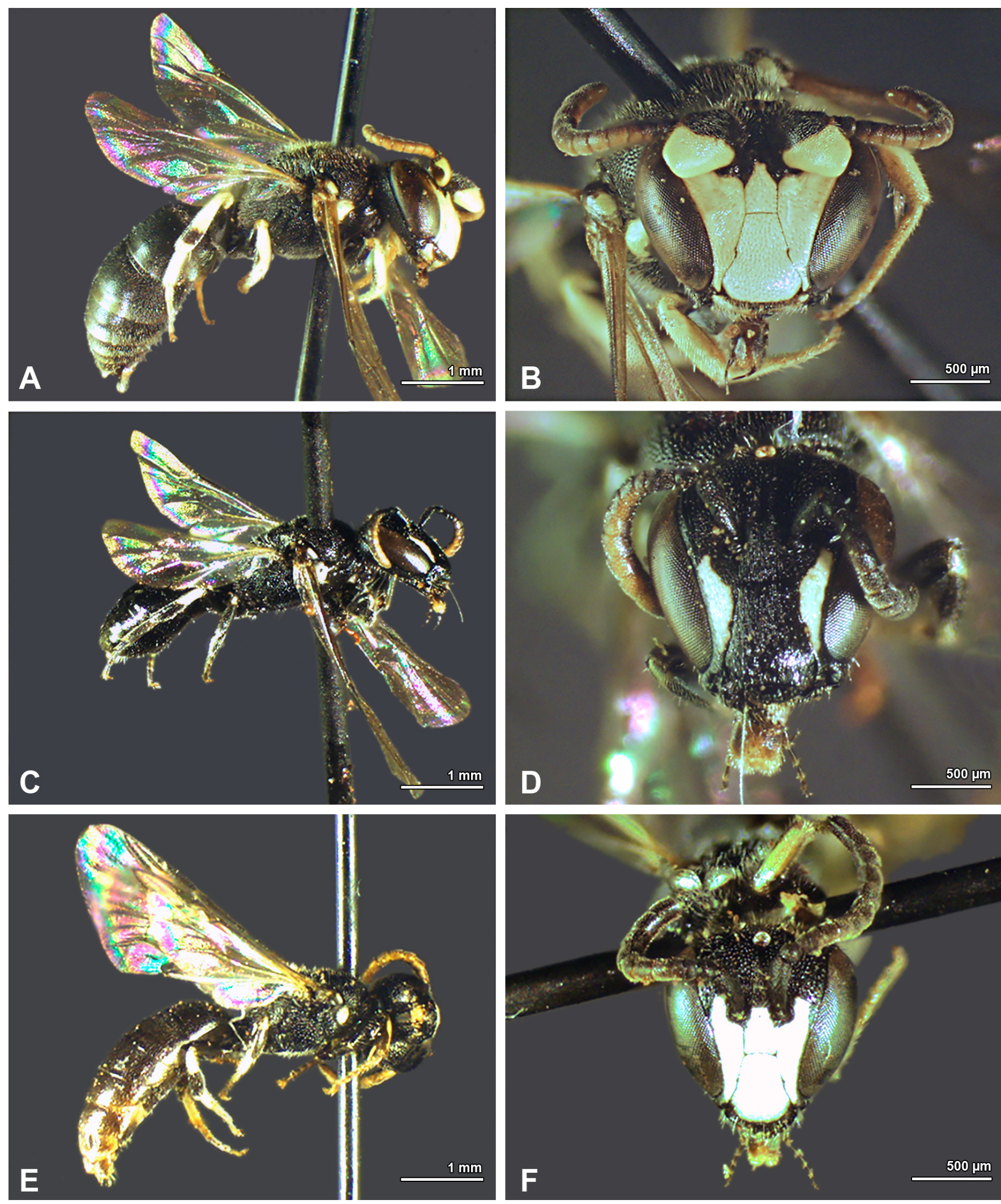

Figure 3. Photographs of Hylaes (Lambdopsis) rinki (Gorski, 1852), H. (Spatulariella) hyalinatus Smith, 1842, H. (Spatulariella) punctatus (Brullé, 1832): A-B. Total body and head in frontal view of H. Rinki, C-D. Total body and head frontal view of H. Hyalinatus, E-F. Total body and head frontal view of H. punctatus. 
The face-sides with no converging impressions, normal and not widened supraclypeal area and the antennal scape which is distinctly longer than wide are the main characteristics of variegatus group (Ozbek \& Dathe, 2020). The species H. lionotus was previously only reported from Central Asia (Kazakhstan). Compared with the specimens from Kazakhstan, in Iranian specimens, the metasoma is not completely red but predominantly black with longer supraclypeal area. These differences may well be due to the morphological variability within this species. However in both Iranian and Kazakhstan samples, the penis valves are completely similar and without any longitudinal edge inside. As the morphological characteristics of this species are not clearly distinguishable from other species of variegatus group, molecular methods could be very informative, particularly to reveal the taxonomic status of the species (Dathe \& Proshchalykin, 2018). Based on Koster (1986), in Netherland, both H. rinki and H. hyalinatus nest on the stems of Rubus sp. and collect pollens of various plants in the family Rosaceae (specially the genus Rubus Linnaeus). Nonetheless in our study, despite the existence of many raspberry bushes (Rosaceae) in the sampling area (Bujan village), both species were collected on Matricaria chamomilla (Asteraceae). Moreover, based on some old literatures (Pittioni, 1952; Koster, 1986), the flight periods of these two species are mid-May to mid-September and mid-May to midOctober, respectively. Nevertheless, our samples were collected in mid-April. The probable reason for this time mismatch could be the climatic differences, as the climate of Iran is influenced by its location between the subtropical aridity of the Arabian Desert areas and the subtropical humidity of the eastern Mediterranean area.

In conclusion, regarding the vastness, climatic diversity and rich and pristine vegetation cover of Iran, the actual number of Colletidae bees, particularly the species of the genus Hylaeus, is expected to be much higher than current reports. Hence, further studies are suggested in this field, particularly in some western and northwestern provinces of the country (Table 1), since so far, no information has been recorded about this group of bees in these regions.

\section{$\%$ of Hylaeus subgenera reported in Iran}

- Spatulariella
- Prospis
- Paragiata
Paraprosopis
- Lambdopsis
Hylaeus
Dentigera
Abrupta

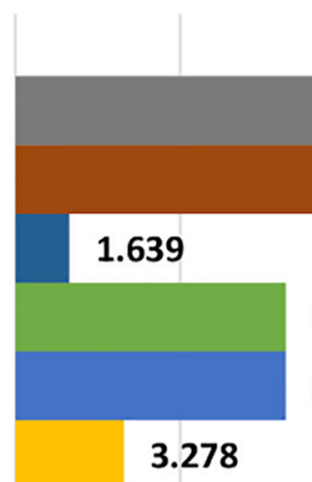

11.475

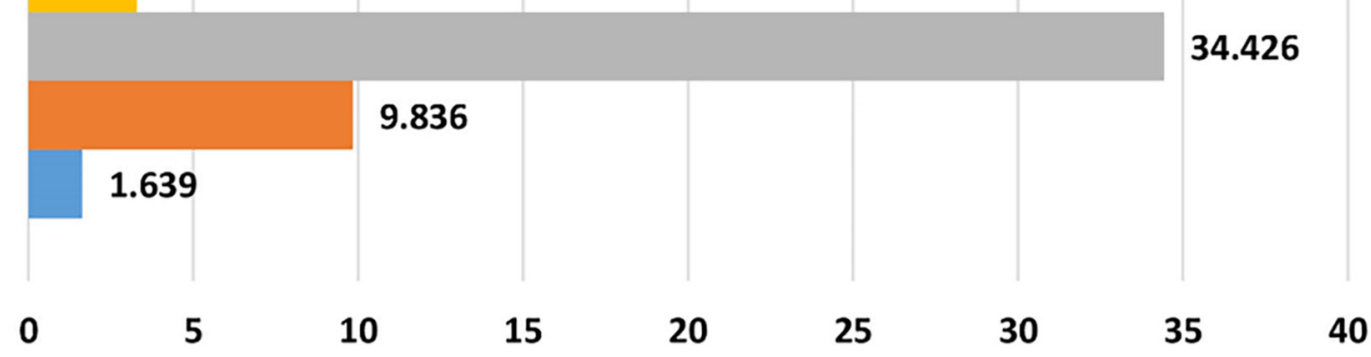

Figure 4. Percentage of the recorded species of Hylaeus subgenera in Iran. 
Table 1. Number of recorded Hylaeus species of each subgenus of the genus Hylaeus in Iranian Provinces.

\begin{tabular}{|c|c|c|c|c|c|c|c|c|c|}
\hline Province & $\frac{\frac{\pi}{2}}{\frac{\pi}{2}}$ & 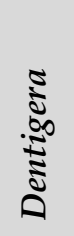 & 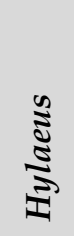 & 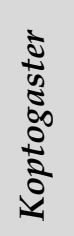 & $\begin{array}{l}\frac{w}{w} \\
\frac{2}{0} \\
\mathbb{0} \\
\vdots \\
5\end{array}$ & $\begin{array}{c}0 \\
\frac{5}{2} \\
\frac{2}{2} \\
0 \\
0 \\
\frac{2}{2} \\
0\end{array}$ & 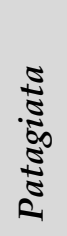 & $\begin{array}{l}\frac{n}{2} \\
\frac{2}{2} \\
0 \\
0 \\
0\end{array}$ & 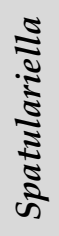 \\
\hline Alborz & - & 1 & 5 & - & 1 & 2 & - & 4 & - \\
\hline Ardabil & - & - & - & - & - & - & - & - & - \\
\hline Bushehr & - & - & - & - & - & 2 & - & 2 & - \\
\hline $\begin{array}{l}\text { Chaharmahal and } \\
\text { Bakhtiari }\end{array}$ & - & - & - & - & - & - & - & - & - \\
\hline East Azarbaijan & - & 1 & - & 1 & - & - & - & 1 & - \\
\hline Fars & - & 2 & 3 & - & - & 1 & - & 3 & 1 \\
\hline Golestan & 1 & 1 & 1 & - & 1 & 1 & 1 & 3 & - \\
\hline Guilan & - & - & - & - & - & - & - & 2 & - \\
\hline Hamedan & 1 & - & 5 & - & - & 1 & - & 3 & 1 \\
\hline Hormozgan & - & - & - & - & - & - & - & 1 & - \\
\hline Ilam & - & - & - & - & - & - & - & - & - \\
\hline Isfahan & 1 & 2 & 11 & - & - & - & - & 2 & - \\
\hline Kerman & - & - & 2 & - & - & 2 & - & 2 & - \\
\hline Kermanshah & - & - & - & - & - & - & - & - & - \\
\hline Khorasan-e Razavi & - & - & 7 & 4 & 1 & - & - & 2 & 2 \\
\hline Khuzestan & 1 & - & - & - & - & 1 & - & 2 & - \\
\hline $\begin{array}{l}\text { Kohgiluye and } \\
\text { Boyerahmad }\end{array}$ & - & - & - & - & - & - & - & - & - \\
\hline Kurdistan & - & - & 1 & - & - & - & - & - & - \\
\hline Lorestan & - & - & - & - & - & - & - & - & - \\
\hline Markazi & - & - & - & - & - & - & - & 1 & - \\
\hline Mazandaran & - & 2 & 4 & 1 & 2 & 1 & - & 5 & 2 \\
\hline North Khorasan & - & - & - & 1 & 2 & - & - & 2 & - \\
\hline Qazvin & - & - & 2 & - & - & - & - & 1 & - \\
\hline Qom & - & - & - & - & - & - & - & - & - \\
\hline Semnan & - & - & - & 1 & - & - & - & 1 & - \\
\hline Sistan and Balouchistan & - & - & 1 & - & - & - & - & 1 & - \\
\hline South Khorasan & - & - & 2 & - & - & - & - & - & - \\
\hline Tehran & - & - & - & - & - & - & - & 1 & 2 \\
\hline West Azarbaijan & - & - & - & - & - & - & - & 1 & - \\
\hline Yazd & - & - & - & - & - & 1 & - & - & - \\
\hline Zanjan & - & - & 1 & - & - & - & - & - & - \\
\hline
\end{tabular}

\section{AUTHOR'S CONTRIBUTION}

The authors confirm contribution to the paper as follows: M.A.: Data collection and species identification; M.A., L.F. and V.G.R.: draft manuscript preparation; all authors approved the final version of the manuscript.

\section{FUNDING}

This work was supported by grants from Ferdowsi University of Mashhad, Iran (Project No. 47906). 


\section{AVAILABILITY OF DATA AND MATERIAL}

Not applicable.

\section{ETHICS APPROVAL AND CONSENT TO PARTICIPATE}

Not applicable.

\section{CONSENT FOR PUBLICATION}

Not applicable.

\section{CONFLICT OF INTERESTS}

The authors declare that there is no conflict of interest regarding the publication of this paper.

\section{ACKNOWLEDGMENTS}

We are grateful to Professor Holger Dathe (Senckenberg Deutsches Entomologisches Institut Senckenberg, Leibniz Institution for Biodiversity and Earth System Research) for his valuable comments.

\section{REFERENCES}

Allahverdi, S., Nadimi, A., Afshari, A. \& Aliyev, K. (2015) A preliminary list of Andrena subgenera (Hymenoptera: Andrenidae) of Iran, with five new records. Journal of Insect Biodiversity and Systematics, 1 (1), 61-75.

Allahverdi, M., Nadimi, A. \& Afshari A. (2016) A survey on family Andrenidae (Hymenoptera: Apoidea) in Gorgan county, Iran. Iranian Journal of Animal Biosystematics, 12 (2), 145-156. https:/ / doi.org/10.22067/ijab.v12i2.51068

Ascher, J.S. \& Pickering, J. (2021) Discover Life bee species guide and world checklist (Hymenoptera: Apoidea). Available from: http:/ / www.discoverlife.org/ [Accessed 21th September 2021].

Arbuckle, T., Schröder, S., Steinhage, V. \& Wittmann, D. (2001) Biodiversity informatics in action: identification and monitoring of bee species using ABIS. In: Proceedings of the 15th International Symposium Informatics for Environmental Protection, 2001, Eidgenössische Technische Hochschule (ETH) University, Zürich, Swetzerland, 1, 425-430.

Celary, W. (1999) New and rare species of the genus Hylaeus Fabricius, 1793 for the fauna of Poland [Hymenoptera: Apoidea: Colletidae]. Acta zoologica cracoviensia, 42 (2), 259-264.

Chen, X. \& Xu, H. (2009) A key to species of the genus Hylaeus (Hymenoptera: Colletidae) from mainland of China with descriptions of new species and new records. Zootaxa, 1974 (1), 31-50. https://doi.org/10.11646/zootaxa.1974.1.3

Dathe, H.H. (2015) Studies on the systematics and taxonomy of the genus Hylaeus F. (9) Supplement to the taxonomy and distribution of Afrotropical Hylaeus F. species (Hymenoptera: Anthophila, Colletidae). Contributions to Entomology, 65 (1), 9-26. https:/ / doi.org/10.21248/contrib.entomol.65.2.223-238

Dathe, H.H. (1980a) Die Hylaeus-Arten einer apidologischen Sammelreise in den Iran (Hymenoptera, Apoidea). Entomologische Abhandlungen Staatliches Museum für Tierkunde in Dresden, 43 (5), 77.

Dathe, H.H. (1980b) Die Arten der Gattung Hylaeus F. in Europa (Hymenoptera: Apoidea, Colletidae). Mitteilungen aus dem Zoologischen Museum in Berlin, 56, 207-294.

Dathe, H.H. \& Proshchalykin, M.Y. (2018) The genus Hylaeus Fabricius in Central Asia (Hymenoptera: Apoidea: Colletidae). Zootaxa, 4517 (1), 1-9. https:// doi.org/10.11646/zootaxa.4517.1.1

Eardley, C.D., Kuhlmann, M. \& Pauly, A. (2010) The bee genera and subgenera of sub-Saharan Africa. ABC Taxa, 7, $1-138$.

Feitz, F., Schneider, N. \& Pauly, A. (2003) Hyménoptères Apocrites nouveaux ou intéressants pour la faune luxembourgeoise (Hymenoptera, Apocrita). Bulletin-Societe des Naturalistes Luxembourgeois, 104, 79-88.

Francisco, F.O., Nunes-Silva. P., Francoy, T.M., Wittmann. D., Imperatriz-Fonseca, V.L., Arias, M.C. \& Morgan, E.D. (2008) Morphometrical, biochemical and molecular tools for assessing biodiversity. An example in Plebeia remota (Holmberg, 1903) (Apidae, Meliponini). Insectes Sociaux, 3, 231-237. https:/ / doi.org/10.1007/s00040-008-0992-7 
Grace, A. (2010) Introductory Biogeography to Bees of the Eastern Mediterranean and Near East. Bexhill, Bexhill Museum. $285 \mathrm{pp}$.

Haris, A. (2015) Preliminary and exploratory investigations of the Aculeata fauna of Danube-Ipoly National Park between 1993 and 1996 (Hymenoptera: Aculeata). Natura Somogyiensis, 27, 75-97. https:// doi.org/10.24394/NatSom.2015.27.75

Huber, F.K., Kaiser, R., Sauter, W. \& Schiestl, F.P. (2005) Floral scent emission and pollinator attraction in two species of Gymnadenia (Orchidaceae). Oecologia, 142 (4), 564-575. https:/ / doi.org/10.1007/s00442-004-1750-9

Izadi, H., Samih, M.A. \& Mahdian, K. (2006) Identification and introduction of some Iran pollinator bees of Colletidae, Halictidae, and Megachilidae (Hym: Apoidea). Communications in Agricultural and Applied Biological Sciences, 71 (2), 621-624.

Kiani Bakiani, S., Monfared, A., Hajiqanbar, H. \& Azhari, S. (2016) A survey on Apoidea (Insecta: Hymenoptera) bees and their associated mites in Fars Province, Iran. Journal of Insect Biodiversity and Systematics, 2 (2), 285-299.

Khaghaninia, S., Güler, Y. \& Dikmen, F. (2013) New records for the bee fauna of Iran (Hymenoptera: Apoidea). Zoology in the Middle East, 59 (4), 319-325. https:/ / doi.org/10.1080/09397140.2013.868134

Khodaparast, R. \& Monfared, A. (2012) A survey of bees (Hymenoptera: Apoidea) from Fars province, Iran. Zootaxa, 3445 (1), 37-58. https:/ / doi.org/10.11646/zootaxa.3445.1.2

Khodarahmi Ghahnavieh, R. \& Monfared, A. (2019) A survey of the bees (Hymenoptera: Apoidea) from Isfahan Province, Iran. Journal of Insect Biodiversity and Systematics, 5 (3), 171-20.

Koster, A. (1986) Het genus Hylaeus in Nederland (Hymenoptera, Colletidae): with a key to the species of NW Europe in english. Zoologische Bijdragen, 36 (1), 3-120.

Kowalczyk, J.K., Kurzac, T. \& Pawlikowski, T. (2009) The state of knowledge on the bees (Hymenoptera, Apoidea, Apiformes) of the Wzniesienia Łódzkie Landscape Park. Parki Narodowe i Rezerwaty Przyrody, 28 (4), 41-55.

Kuhlmann, M. (2005) Diversity, distribution patterns and endemism of southern African bees (Hymenoptera: Apoidea). African Biodiversity, 29, 167-172. https://doi.org/10.1007/0-387-24320-8_13

Michener, C.D. (2007) The Bees of the World. Second edition. Baltimore: Johns Hopkins University Press. 953 pp.

Müller, A., Diener, S., Schnyder, S., Stutz, K., Sedivy, C. \& Dorn, S. (2006) Quantitative pollen requirements of solitary bees: implications for bee conservation and the evolution of bee-flower relationships. Biological Conservation, 130 (4), 604-615. https:/ / doi.org/10.1016/j.biocon.2006.01.023

Nadimi, A., Talebi, A.A. \& Fathipour, Y. (2013a) A preliminary study of the cleptoparasitic bees of the genus Coelioxys (Hymenoptera: Megachilidae) in northern Iran, with six new records. Journal of Crop Protection, 2 (3), 271-283.

Nadimi, A., Talebi, A.A. \& Fathipour, Y. (2013b) The tribe Osmiini (Hymenoptera: Megachilidae) in the north of Iran: new records and distributional data. Entomofauna, 34 (17), 205-220.

Nadimi, A., Talebi, A.A. \& Fathipour, Y. (2014) Study of the tribe Anthidiini (Hymenoptera: Megachilidae) in northern Iran, with the description of a new species. North-Western Journal of Zoology, 10, 413-424.

Nazari, S., Monfared, A., Nemati, A. \& Azhari, S. (2019) A survey on bees (Insecta, Hymenoptera, Apoidea) and their associated mites in Chaharmahal and Bakhtiari province of Iran. Journal of Insect Biodiversity and Systematics, 5 (2), 107-120.

Özbek, H. \& Dathe, H.H. (2020) The bees of the genus Hylaeus Fabricius, 1793 of Turkey, with keys to the subgenera and species (Hymenoptera: Anthophila, Colletidae). Contributions to Entomology, 70 (2), 273-346. https:// doi.org/10.21248/contrib.entomol.70.2.273-346

Pittioni, B. (1952) Über Variabilität und Verbreitung der westpaläarktischen Arten der Gattung Spatulariella Pop. (Hym., Apoidea, Colletidae). Zeitschrift der Wiener Entomologischen Gesellschaft, 63, 187-204.

Popov, V.B. (1967) The bees (Hymenoptera: Apoidea) of Iran. Trudy Zoologicheskogo Instituta Leningrad, 43, 184-215.

Proshchalykin, M.Y. \& Dathe, H.H. (2016) Additional records of the genus Hylaeus Fabricius, 1793 (Hymenoptera: Apoidea: Colletidae) from Siberia, with description of a new species. Zootaxa, 4105 (4), 301-320.

https://doi.org/10.11646/zootaxa.4105.4.1 
Proshchalykin, M.Y. \& Dathe, H.H. (2012). The bees of the genus Hylaeus Fabricius 1793 of the Asian part of Russia, with a key to species (Hymenoptera: Apoidea: Colletidae). Zootaxa, 3401 (1), 1-36.

https://doi.org/10.11646/zootaxa.3401.1.1

Proshchalykin, M.Y. \& Dathe, H.H. (2021) New and little-known bees of the genus Hylaeus Fabricius, 1793 (Hymenoptera, Colletidae) from the Caucasus region. Journal of Hymenoptera Research, 84, 169-185. https:// doi.org/10.3897/jhr.84.68250

Radchenko, V.G. (1996) Evolution of nest building in bees (Hymenoptera, Apoidea). Entomological Review, 75 (6), 20-32.

Radchenko, V.G. (2017) A new bee species of the genus Dasypoda Latreille (Hymenoptera, Apoidea) from Portugal with comparative remarks on the subgenus Heterodasypoda Michez. Zootaxa, 4350 (1), 164-176. https:// doi.org/10.11646/zootaxa.4350.1.10

Radchenko, V.G. \& Pesenko, Y.A. (1994) Biology of bees (Hymenoptera, Apoidea). Russian Academy of Science Zoological Institute, St Petersburg, 6, 313-331

Radchenko, V.G. \& Pesenko, Y.A. (1996) " Protobee" and its nests: a new hypothesis concerning the early evolution of Apoidea (Hymenoptera). Entomological Review, 75 (2), 140-162.

Safi, Z., Nadimi, A., Yazdanian, M. \& Radchenko, V.G. (2018) Report of one rare bee new to Iran, with the checklist of the Persian bee subfamily Nomioidinae (Hymenoptera: Halictidae). North-Western Journal of Zoology, 14 (2), 159-164.

Salarian, M., Nadimi, A., Talebi, A.A., Radchenko, V.G. (2016) A survey of the genus Ceratina Latreille (Hymenoptera: Apidae) in northern Iran, with three new records. Journal of Insect Biodiversity and Systematics, 2 (1), 143-154.

Sárospataki, M., Novák, J. \& Molnár, V. (2005) Assessing the threatened status of bumble bee species (Hymenoptera: Apidae) in Hungary, Central Europe. Biodiversity \& Conservation, 14 (10), 2437-2446. https://doi.org/10.1007/s10531-004-0152-y

Steffan-Dewenter, I. \& Tscharntke, T. (2001) Succession of bee communities on Fallows. Ecography, 24, 83-93. https://doi.org/10.1034/j.1600-0587.2001.240110.x

Warncke, K. (1981) Beitrag zur Bienenfauna des Iran 12. Die Gattung Prosopis F. mit Bemerkungen zu weiteren bekannten und unbekannten paläarktischen Arten. Bollettino Del Museo Civico di Storia Naturale di Venezia, 31, 145-195. [in German]

Zhou, Q.S., Luo, A., Zhang, F., Niu, Z.Q., Wu, Q.T., Xiong, M., Orr, M.C. \& Zhu, C.D. (2020) The first draft genome of the plasterer bee Colletes gigas (Hymenoptera: Colletidae: Colletes). Genome Biology and Evolution, 12 (6), 860 866. https://doi.org/10.1093/gbe/evaa090 
(Hymenoptera: Apoidea: Colletidae) Hylaeus Fabricious, 1793 فهرست زنبورهاى كردهافشان جنس در ايران

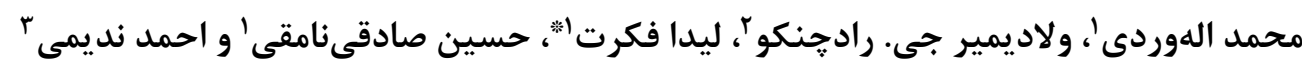

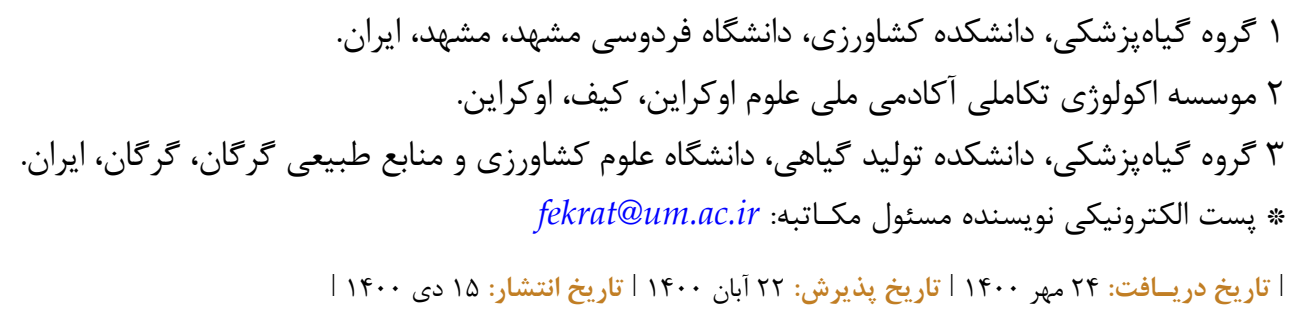

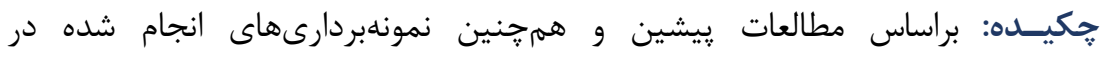

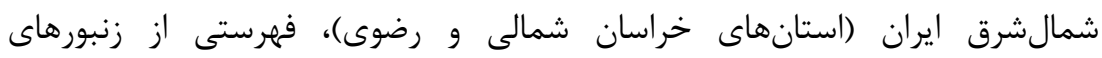

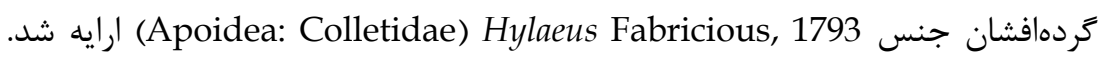

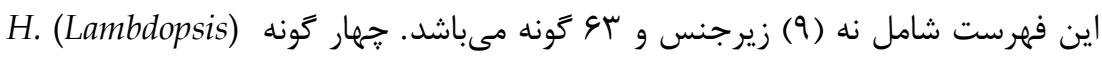
،H. (Spatulariella) hyalinatus Smith, 1842 ،rinki (Gorski, 1852) H. lionotus (Alfken, 1909) g H. (Spatulariella) punctatus (Brullé, 1832)

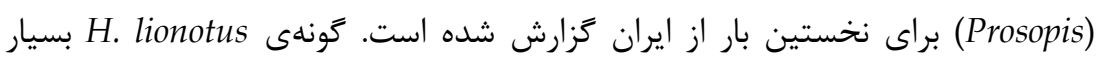

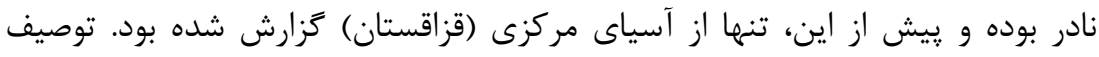

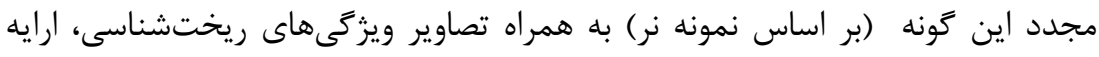

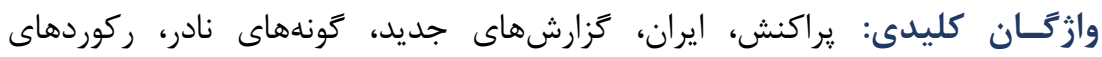

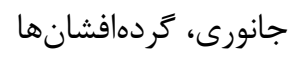

